УдК 621.37

\title{
ВОЗБУЖДЕНИЕ ОДНОРОДНОЙ ИЗОТРОПНОЙ СРЕДЫ СКАЧКОМ МАГНИТНОГО ПОЛЯ
}

НАЙДЕНКО В. И.

Национальный технический университет Украины

«Киевский политехнический институт»,

Украина, Киев, 03056, пр-т Победь 37

\begin{abstract}
Аннотация. В замкнутом виде решена задача возбуждения однородной изотропной среды скачком Хэвисайда магнитного поля. Приведены результаты расчетов поля в среде
\end{abstract}

Ключевые слова: импульс Гаусса; функция Хэвисайда; преобразование Лапласа; уравнения Максвелла

\section{ВВЕДЕНИЕ}

Задачи распространения электромагнитных волн с аналитическим решением имеют важное значение. Они позволяют точно определить фундаментальные характеристики электромагнитного поля. К сожалению, таких задач мало и они в основном решены. Как правило, рассматривается задача возбуждения сглаженными сигналами, например импульсом Гаусса [1]. При этом нивелируется влияние высших гармоник в разложении поля в ряд Фурье. Подобная задача возбуждения среды рассмотрена в [2]. Правильный результат получено при условии, что среда является проводником. Это допущение значительно упрощает задачу. Однако в этом случае невозможно определить влияние тока смещения, который не учитывается уже на этапе постановки задачи. Такой подход обусловлен наличием в литературе соответствующего обратного преобразования Лапласа.

К классу задач, которые имеют аналитическое решение, принадлежат задачи возбуждения среды импульсным (не сглаженным) сиг- налом. Данная статья посвящена решению задачи возбуждения среды скачком магнитного поля. Она начинает серию работ, которые касаются возбуждения полей не сглаженными сигналами. Среди них решение задачи возбуждения полей сигналами в виде одиночного импульса, что открывает возможность исследования распространения сверхкоротких импульсов и быстроизменяющихся сигналов, что важно для перспективных радаров, электромагнитных снарядов и т.п. [3].

\section{ПОСТАНОВКА ЗАДАЧИ}

В среде с параметрами $\varepsilon_{0} \varepsilon, \mu_{0} \mu, \sigma$ в момент $t=0$ в плоскости $z=0$ внешним источником скачком создается магнитное поле по направлению вдоль оси $y$, напряженность которого $H_{y 0}(t)=H_{0} \eta(t)$, где $\eta(t)-$ единичная функция (функция включения Хэвисайда):

$$
\eta(t)= \begin{cases}0, & t<0 \\ 1, & t>0\end{cases}
$$

Созданное магнитное поле не зависит от поперечных координат $x$ и $y$. Необходимо най-

DOI: $10.20535 /$ S0021347016060078

() Найденко В. И., 2016 